\title{
Ruptured Aortic Aneurysm and Dissection Related Death: an Autopsy Database Analysis
}

\author{
Dániel Pál ${ }^{1} \cdot$ Brigitta Szilágyi ${ }^{2}$ : Márton Berczeli ${ }^{1} \cdot$ Csaba Imre Szalay ${ }^{3} \cdot$ Balázs Sárdy $^{1} \cdot$ Zoltán Oláh $^{1} \cdot$ Tamás Székely $^{4}$. \\ Gergely Rácz ${ }^{5}$ Péter Banga ${ }^{1} \cdot$ Zsófia Czinege $^{1} \cdot$ Péter Sótonyi $^{1}$
}

Received: 22 March 2020 / Revised: 22 March 2020 / Accepted: 27 May 2020 / Published online: 16 June 2020

(C) The Author(s) 2020

\begin{abstract}
Acute aortic catastrophes (AAC), mainly ruptured aneurysms and dissections, lead all other vascular conditions in morbidity and mortality, even if intervention occurs. The aim of our study was to give a descriptive overview of the demographic and pathological characteristics of AAC. Between 1994 and 2013, 80,469 autopsies were performed at Semmelweis University hospitals in Budapest. After collecting the autopsy reports we were able to create the AAC database upon which we conducted our analysis. We found 567 cases of AAC. The cause of death in 120 of them was classified as a non-ruptured aorta with malperfusion or distal embolization. Of the remaining 447 cases, in 305 the cause of death was a ruptured aortic aneurysm (rAA), and in 142 it was a ruptured aortic dissection (rAD). The distribution of rAA cases was $34.4 \%$ thoracal, $4.3 \%$ thoracoabdominal, and $61.3 \%$ abdominal. We found female dominance where the rAA was thoracal. In rAD cases, $84 \%$ were Stanford A and $16 \%$ Stanford B type. In both groups we found different pathological distributions. In the prehospital group, the number of thoracal ruptures was considerable. $88 \%$ of the patients with Stanford A dissection died in the prehospital or perioperative period. The most progressive AACs were ruptures of intrapericardial aneurysms and Stanford A dissections., however survival rate can be elevated by using rapid imaging examination and immediate surgical intervention. We want to highlight that our study contains such gender differences, which are worth to be taken into consideration.
\end{abstract}

Keywords Acute aortic syndrome $\cdot$ Aortic dissection $\cdot$ Aortic aneurysm $\cdot$ Autopsy $\cdot$ Rupture $\cdot$ Bleeding

\begin{abstract}
Abbreviations
AAC Acute aortic catastrophes

AAA Abdominal aortic aneurysm

rAA Ruptured aortic aneurysm

rAD Ruptured aortic dissection
\end{abstract}

Péter Sótonyi

sotonyi.peter1@med.semmelweis-univ.hu; sotonyi@hotmail.com

1 Heart and Vascular Center, Department of Vascular and Endovascular Surgery, Semmelweis University, Városmajor u. 68, Budapest 1122, Hungary

2 Department of Geometry, Institute of Mathematics, Budapest University of Technology and Economics, Budapest, Hungary

3 Heart and Vascular Center, Department of Cardiology, Semmelweis University, Budapest, Hungary

4 2nd Department of Pathology, Semmelweis University, Budapest, Hungary

5 1st Department of Pathology and Experimental Cancer Research, Semmelweis University, Budapest, Hungary
TAA Thoracal aortic aneurysm

ThAA Thoracoabdominal aortic aneurysm

rAAC Ruptured acute aortic catastrophe

nrAAC Non-ruptured acute aortic catastrophe

\section{Introduction}

Cardiovascular diseases are the leading cause of death in Hungary [1]. Among them, AACs caused by aortic rupture are relatively rare, but they are severe conditions with high mortality. Most frequently, AACs are caused by rAA and rAD.

Aortic aneurysms form in both the abdominal and thoracal segments. There are gender differences recorded in the literature $[2,3]$, which we consider in our analysis. Aortic aneurysm formation occurs most frequently in the abdominal segment - almost 9 times more frequently than in the thoracic segment [4]. According to multiple studies, the prevalence of abdominal aortic aneurysm (AAA) ranges from $1.3-12.7 \%$ in 
the elderly population, with a male to female ratio of (2-4):1 [5-7]. The incidence of ruptured AAAs is $1-21$ cases per 100,000 persons/year, with a male-female ratio of (6-9):1 $[6,8]$. They are not rare, however, and show an increasing incidence, 59 to 104 cases per 1,000,000 persons/year [9, 10]. Nowadays, screening for AAAs is recommended for high-risk groups such as male smokers over 65 [11]. The distribution of thoracal aortic aneurysms TAAs is as follows: $40 \%$ on the ascending segment, $15 \%$ on the aortic arch, $35 \%$ on the descending segment, and $10 \%$ are thoracoabdominal aortic aneurysms ThAAs [10]. Early diagnosis of aortic aneurysms is crucial for successful treatment.

Acute aortic dissection (AAD) is one of the most common catastrophic events affecting the aorta, with an incidence of $(29-35) / 1,000,000$ persons/year $[12,13]$. The intimal tear is located in the ascending aorta in $65 \%$ of the cases, the descending aorta in $25 \%$, and in the arch or the abdominal aorta in 10\% [14]. The incidence of Stanford A and B type dissections peaks at 50 and 60 years, respectively. The male-tofemale ratio has been reported to be 4:1 $[14,15]$. Depending on the affected segment, the most common complications are aortic rupture, cardiac tamponade, aortic regurgitation, thromboembolism, and the compression of coronary or peripheral arteries $[16,17]$. The diagnosis of AAD is difficult because the possible symptoms can mislead the physician to other kinds of pathologies, such as myocardial infarction or stroke. The mortality rate of hospitalized but untreated Stanford A dissection patients can be as high as 50-58\% [14, 18].

The aim of our research was to investigate the occurrence of aortic ruptures leading to death using the medical autopsy records of the institutes of Semmelweis University, to describe the most specific clinicopathological parameters of AAC, and to investigate certain demographic and morphological features regarding cases with aortic aneurysm rupture or acute dissection leading to rupture.

\section{Materials and Methods}

We collected autopsy data from the records of Semmelweis University's (Budapest, Hungary) 1st Department of Pathology and Experimental Cancer Research, 2nd Department of Pathology, and the Department of Forensic and Insurance Medicine for the 20-year period from 1994.01.01 to 2013.12.31. These institutes perform autopsies in cases when sudden death occurs in public institutions and public spaces in the entire area of Budapest and Pest County, as well as on the premises of Semmelweis University. Budapest and Pest County are home to about $25 \%$ of the Hungarian population [19]. During the 20-year period, 80,469 autopsies were reported. We analyzed every case where the "cause of death" or the "diagnosis related to the patient's death" was defined as aortic aneurysm or aortic dissection. We collected these cases using the International Classification of Diseases, Tenth Revision, Clinical Modification (ICD-10-CM) code (aortic dissection: I7100I7103 and aortic aneurysm: I711-I718).

Based on the resulting list, we checked the medical records for each case and created a database with the following data:

- Demographics: age, gender, hospital-admission information, date and place of death,

- Type of aortic pathology: aortic aneurysm (AA) or aortic dissection $(\mathrm{AD})$,

- Localization of the lesion: ascending, arch, descending, thoracoabdominal, suprarenal, infrarenal, or combined,

- Extent of the aortic dissection: Stanford A or B type, and

- Direction of the bleeding (if rupture occurred): thorax, pericardium, bronchi, esophagus, abdominal cavity, cava, or retroperitoneum.

Then we organized the population into groups and analyzed the data. We divided the total population into three groups according to hospital admission and therapeutic intervention: Group I suffered pre-hospital death, group II suffered preintervention hospital death, and group III suffered peri- or post-intervention death. We also divided the population into two groups according to the two major complications of AAC that lead to death: ruptured acute aortic catastrophe (rAAC) or non-ruptured acute aortic catastrophe (nrAAC). Finally, we analyzed the yearly and monthly distribution of cases, the demographic data, the clinical course of each case, the place of death (home, public space, ambulance, or hospital), and the place of medical care (emergency department, intensive care unit, general surgery department, or cardiovascular surgery department).

\section{Results}

Of the 80,469 recorded autopsies, aortic aneurysm or dissection was the cause of death in $567(0.7 \%)$ cases. Of these 567 AAC cases, 447 (79\%) belong in the rAAC group, while 120 $(21 \%)$ in the nrAAC group. In the nrAAC group, the aortic event leads indirectly to the patient's death (malperfusion by intimal flap or distal embolization - stroke, mesenteric ischemia, myocardial infarction, respiratory insufficiency, acute renal failure, etc.). The rAAC group, where the aortic bleeding leads directly to the patient's death, is further divided into two subgroups: ruptured aortic aneurysm (rAA) in 305 cases $(68.2 \%)$ and ruptured aortic dissection (rAD) in 142 cases (31.8\%) (Fig. 1).

\section{Non-ruptured AAC Group}

Two thirds of the lethal complications occurred in the nrAD group. The most common causes of death were acute heart 
Fig. 1 Acute aortic catastrophes, $1994-2014, \mathrm{~N}=567$

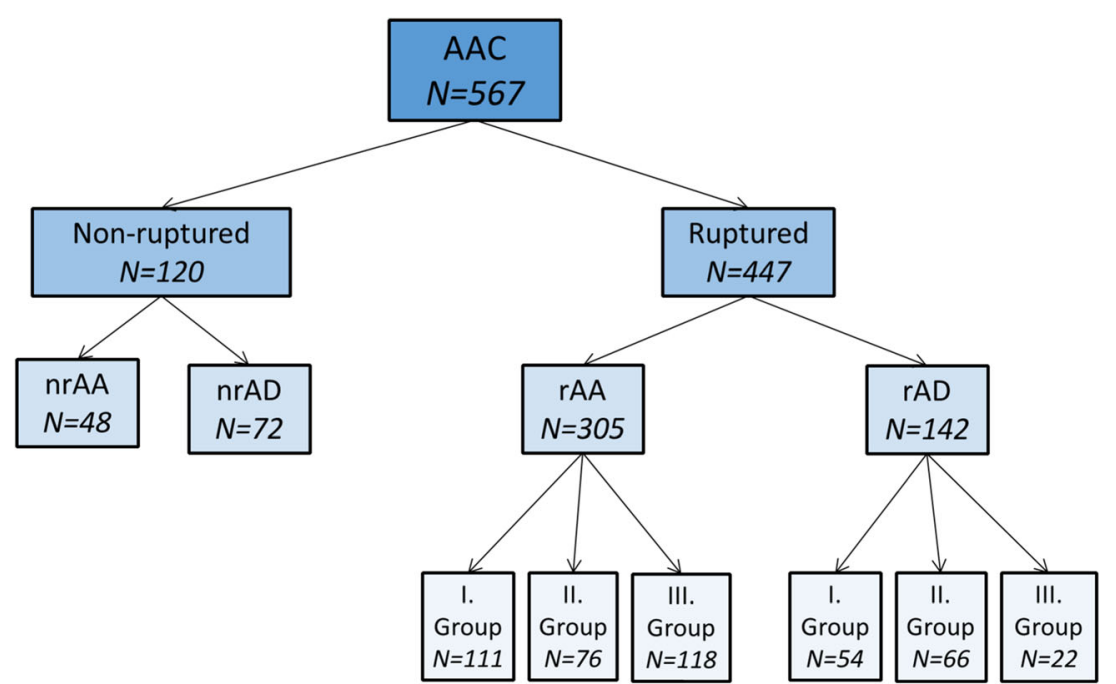

failure, acute mesenteric ischemia, and preoperative stroke. In 56 cases ( $47 \%$ of the nrAAC group), we found distal embolization. We defined malperfusion syndrome as a complication caused by branch-vessel involvement and resulting in endorgan ischemic dysfunction by folating intimal flap in 64 cases (53\% of the nrAAC group). This was verified by pathological examination (macroscopic and histological) (Table 1).

\section{Ruptured AAC Group}

Patients who died as a result of rupture and congestive bleeding form a homogenous and large-scale population. We focused on this group and eanalyzed 447 ruptured cases. In the examined institutes of Semmelweis University, the yearly

Table 1 Analysis of non-ruptured AAC cases $(\mathrm{N}=120)$

\begin{tabular}{lll}
\hline Distal occlusions (N=120) & N & Total (\%) \\
Coronary & 9 & 8 \\
Supraaortic branches & 6 & 5 \\
Visceral branches & 24 & 20 \\
Lower limb artery & 8 & 7 \\
Combination & 9 & 8 \\
Occlusion by intimal flap & 64 & 53 \\
Cause of death (N=120) & N & Total (\%) \\
Acute heart failure, acute myocardial infarction & 61 & 51 \\
Hemorrhagic shock, consumption coagulopathy & 3 & 3 \\
Acute ischaemic colitis, peritonitis & 13 & 11 \\
Acute renal failure & 1 & 1 \\
Pulmonary embolism, ARDS & 5 & 4 \\
Stroke & 9 & 8 \\
Intraoperative, irreversible, acute circulatory failure & 11 & 9 \\
Postoperative sepsis & 12 & 10 \\
Postoperative, irreversible circulatory failure, ARDS & 5 & 4 \\
\hline
\end{tabular}

average of the autopsy cases were: 15 for rAA and 7 for rAD. During our twenty years-long followup, the number of cases showed an increasing tendency, while the overall number of autopsies remained unchanged. There was an increase in the rAA group, from 3.27 to 4.29 of 1,000 autopsies. In the rAD group autopsies also increased, from 1.42 to 2.02 of 1,000 .

\section{Demographic Data}

The average age at the time of death was $71.0 \pm 13.1$ years in the rAA group. In the rAD group, it was $62.6 \pm 14.5$ years. The male/female distribution in the rAA group was 21:10 and $3: 2$ in the rAD group. The proportion of female pacients increased in older age groups. At the second decade of our examined period, average age increased from $70.0 \pm 11.8$ $(\mathrm{N}=126)$ to $71.7 \pm 13.9(\mathrm{~N}=179)$ in the rAA group, while in the rAD group, it decreased from $63.8 \pm 14.8(\mathrm{~N}=56)$ to $62.4 \pm 14.4(\mathrm{~N}=83)$. The youngest patient with rAA was 28 years old, while at the case of $\mathrm{rAD}$ the youngest patient was 14 , futhermore the oldest patient with rAA was 100 years old and with $\mathrm{rAD}$ the oldest one was 94 (Fig. 2a and b).

\section{Clinicopathology}

Regarding the localization of the aortic aneurysms, the autopsy reports showed that $61.3 \%$ of the aneurysms were abdominal, $34.4 \%$ were thoracic and $4.3 \%$ were thoracoabdominal. As we disaggregated the cases by gender, we found a difference. In men, there was a balanced proportion of all three regions, while for women the ascending region dominated. Chest location was significantly higher among women (total chest lesions in females were $46 \%$ and males 28.8\%) (Fig. 3a and $b$ )

In the rAD group, the rate of Stanford A type cases was much higher than Stanford B types. Thoracic aneurysms and 
Fig. 2 a Age and gender differencies in rAA group, 1994 2014, N=305. b Age and gender differencies in rAD group, 19942014, N=142
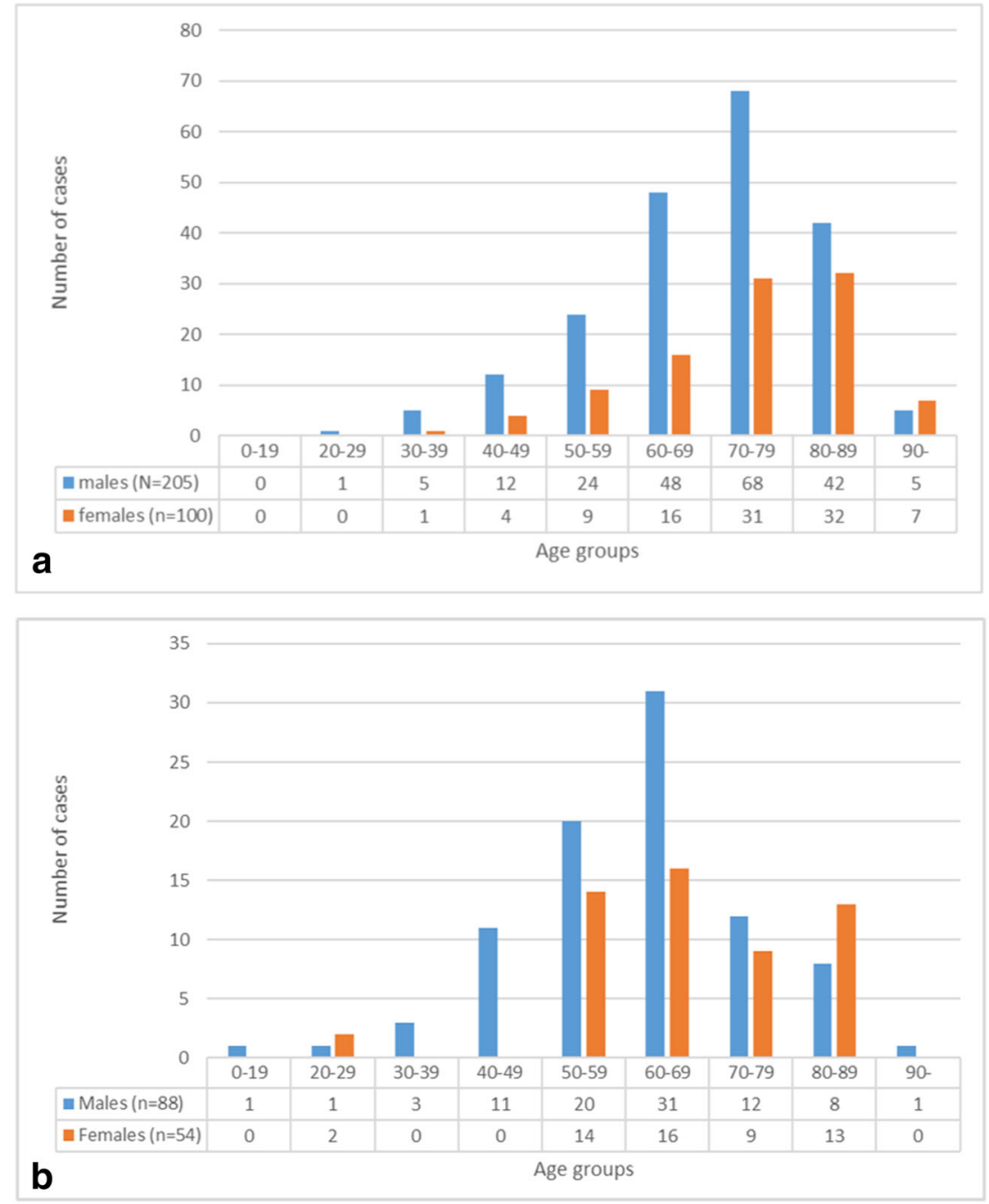

the Stanford A dissections predominantly bled in the direction of the pericardium or the thoracic cavity. We did not find localization or gender difference in the rAD group (Table 2).

\section{Clinical Course}

Analyzing the place of death revealed that patients in both groups had a similar chance of admission into a health care institute $63.6 \%$ for rAA $(\mathrm{N}=194)$ and $62.0 \%$ for $\mathrm{rAD}(\mathrm{N}=$ 88 ). For the rest of the patients, death was diagnosed in public spaces, at home (rAA $28.9 \%$, rAD $32.4 \%$ ), or during transportation to a health care institute (rAA 7.5\%, rAD 5.6\%).

Among those cases in which the death occured in a health care institute, $70.6 \%$ of rAA cases reached the cardiovascular surgery department, $15.5 \%$ reached the emergency department, $9.8 \%$ were in other surgical departments, and $4.1 \%$ were in the intensive care unit. $52.3 \%$ of the hospitalized rAD cases reached the cardiovascular surgery department, $34.1 \%$ reached the emergency department, $5.7 \%$ were in other surgical departments, and $8.0 \%$ were in the intensive care unit.
Based on the place of death we created three groups. then we conducted a more detailed pathological analysis of these groups. In the prehospital group (Group I), the largest number of the cases was retroperitoneal, but the case number of ascending aorta ruptures were also considerable ( $13 \%$ out of 305 cases). In the perioperative (Group II) and postoperative (Group III) groups, the direction of bleeding was retroperitoneal in almost one third of the cases (32\%) (Table 3).

$74 \%$ of the Stanford A type rADs were found in the prehospital and perioperative groups. The most frequent direction of rupture bleeding was pericardial, and over one third of these patients died before receiving medical attention, while another third of them died before adequate surgical intervention could have been performed (Table 4).

\section{Discussion}

A large number of studies focus on AACs, but most of these concentrate on the diagnosis and treatment. Meanwhile, our 
Fig. 3 a Gender differencies in rAA localisation, 1994-2014, $\mathrm{N}=305$. b Gender differences in direct of rupture, rAA group, 1994-2014, N=305
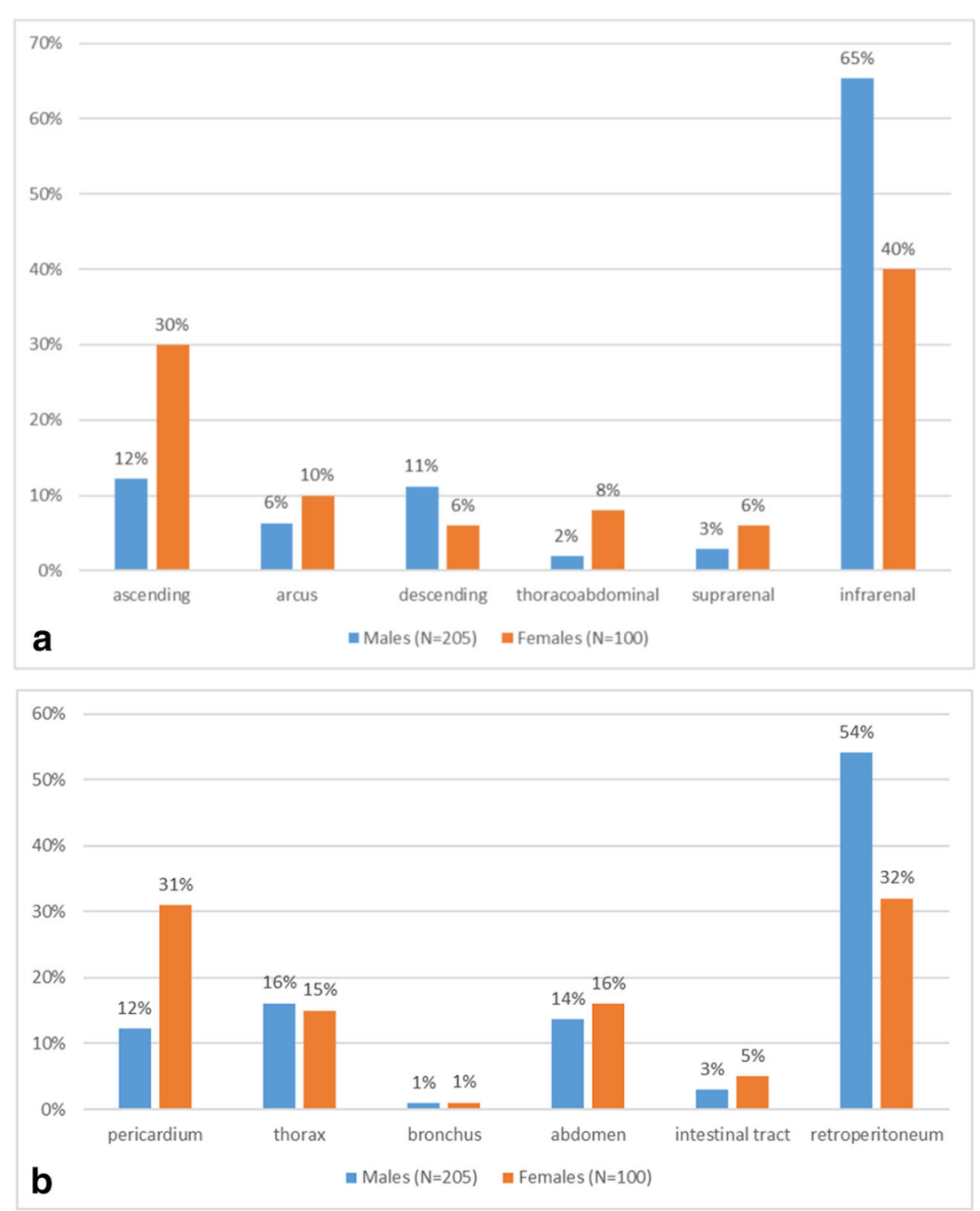

aim was to describe the most important clinicopathological features of these high mortality disorders. In formerly published literature, we found a similar publication [20]; however, a clear comparison cannot be made, due to changes in
Table 2 rAD clinicopathology, 1994-2014 ( $\mathrm{N}=142)$

\begin{tabular}{|c|c|c|c|c|c|c|}
\hline Types & \multicolumn{6}{|c|}{ Direction of rupture } \\
\hline \multicolumn{7}{|c|}{ rAD cases $(\mathrm{N}=142)$} \\
\hline & Bronchus & Thorax & Pericardium & Abdomen & Retroperitoneum & $\operatorname{Sum}(\%)$ \\
\hline A & 0 & 12 & 107 & 0 & 0 & $119(84)$ \\
\hline B & 1 & 11 & 4 & 2 & 5 & $23(16)$ \\
\hline Sum $(\%)$ & $1(1)$ & $23(16)$ & $111(78)$ & $2(1)$ & $5(4)$ & \\
\hline \multicolumn{7}{|c|}{ Males (N=88) } \\
\hline A & 0 & 8 & 66 & 0 & 0 & $74(84)$ \\
\hline B & 0 & 5 & 3 & 1 & 5 & $14(16)$ \\
\hline Sum $(\%)$ & $0(0)$ & $13(15)$ & $69(78)$ & $1(1)$ & $5(6)$ & \\
\hline \multicolumn{7}{|c|}{ Females $(\mathrm{N}=54)$} \\
\hline A & 0 & 4 & 41 & 0 & 0 & $45(83)$ \\
\hline B & 1 & 6 & 1 & 1 & 0 & $9(17)$ \\
\hline Sum $(\%)$ & $1(2)$ & $10(19)$ & $42(78)$ & $1(2)$ & $0(0)$ & \\
\hline
\end{tabular}


Table 3 Pathological differences depending on the place of death, rAA group, 1994-2014 (N=305)

The location of the rAA

\begin{tabular}{|c|c|c|c|c|c|c|c|}
\hline & \multicolumn{2}{|l|}{ I. group } & \multicolumn{2}{|c|}{ II. group } & \multicolumn{2}{|l|}{ III. group } & \multirow{2}{*}{$\begin{array}{l}\text { Sum } \\
\text { N (\%) }\end{array}$} \\
\hline & $\mathrm{N}(\%)$ & in subtype \% & $\mathrm{N}(\%)$ & in subtype \% & $\mathrm{N}(\%)$ & in subtype \% & \\
\hline Ascending & $39(13)$ & 71 & $9(3)$ & 16 & $7(2)$ & 13 & $55(18)$ \\
\hline Arcus & $9(3)$ & 41 & $7(2)$ & 32 & $6(2)$ & 27 & $22(7)$ \\
\hline Descending & $8(3)$ & 29 & $7(2)$ & 25 & $13(4)$ & 46 & $28(9)$ \\
\hline Thoracoabdominal & $1(0)$ & 8 & $5(2)$ & 38 & $7(2)$ & 54 & $13(4)$ \\
\hline Suprarenal & $4(1)$ & 33 & $3(1)$ & 25 & $5(2)$ & 42 & $12(4)$ \\
\hline Infrarenal & $50(15)$ & 29 & $45(14)$ & 27 & $80(23)$ & 44 & $175(57)$ \\
\hline Sum & $111(36)$ & & $76(25)$ & & $118(39)$ & & \\
\hline \multicolumn{8}{|c|}{ The direction of the rupture } \\
\hline Pericardium & $41(13)$ & 73 & $10(3)$ & 18 & $5(2)$ & 9 & $56(18)$ \\
\hline Thorax & $13(4)$ & 27 & $11(4)$ & 22 & $25(8)$ & 51 & $49(16)$ \\
\hline Bronchus & $2(1)$ & 67 & $1(0)$ & 33 & $0(0)$ & 0 & $3(1)$ \\
\hline Retroperitoneum & $42(14)$ & 30 & $36(12)$ & 26 & $63(21)$ & 45 & $141(46)$ \\
\hline Abdomen & $13(4)$ & 29 & $14(5)$ & 31 & $18(6)$ & 40 & $45(15)$ \\
\hline Intestinal & $0(0)$ & 0 & $4(1)$ & 36 & $7(2)$ & 64 & $11(4)$ \\
\hline Sum & $111(36)$ & & $76(25)$ & & 118 (39) & & \\
\hline
\end{tabular}

I. group: prehospital cases $(\mathrm{N}=111)$

II. group: preoperative cases $(\mathrm{N}=76)$

III. group: perioperative and postoperative cases $(\mathrm{N}=118)$

understanding, classification, historical and demographic aspects, as well as changes in the regulations concerning autopsies.
Worldwide, approximately $0.5 \%$ of all deaths is caused by aortic aneurysm and dissection, and according to some research, the proportion may increase [21]. Our findings reveal

Table 4 Pathological differences depending on the place of death, rAD group, 1994-2014, (N=142)

The location of the rAD

\begin{tabular}{|c|c|c|c|c|c|c|c|}
\hline & \multicolumn{2}{|l|}{ I. group } & \multicolumn{2}{|c|}{ II. group } & \multicolumn{2}{|c|}{ III. group } & \multirow[b]{2}{*}{$\operatorname{Sum}(\%)$} \\
\hline & $\mathrm{N}(\%)$ & in subtype $\%$ & $\mathrm{~N}(\%)$ & in subtype $\%$ & $\mathrm{~N}(\%)$ & in subtype $\%$ & \\
\hline Stanford A & $48(34)$ & 40 & $57(40)$ & 48 & $14(10)$ & 12 & $119(84)$ \\
\hline Stanford B & $6(4)$ & 26 & $9(6)$ & 39 & $8(6)$ & 35 & $23(16)$ \\
\hline $\operatorname{Sum}(\%)$ & $54(38)$ & & $66(46)$ & & $22(15)$ & & \\
\hline \multicolumn{8}{|c|}{ The direction of the rupture } \\
\hline Pericardium & $50(35)$ & 45 & $50(35)$ & 45 & $11(8)$ & 10 & $111(78)$ \\
\hline Thorax & $2(1)$ & 9 & $13(9)$ & 57 & $8(6)$ & 35 & $23(16)$ \\
\hline Bronchus & $0(0)$ & 0 & $1(1)$ & 100 & $0(0)$ & 0 & $1(1)$ \\
\hline Retroperitoneum & $1(1)$ & 20 & $1(1)$ & 20 & $3(2)$ & 60 & $5(4)$ \\
\hline Abdomen & $1(1)$ & 50 & $1(1)$ & 50 & $0(0)$ & 0 & $2(1)$ \\
\hline $\operatorname{Sum}(\%)$ & $54(38)$ & & $66(46)$ & & $22(15)$ & & \\
\hline
\end{tabular}

I. group: prehospital cases $(\mathrm{N}=54)$

II. group: preoperative cases $(\mathrm{N}=66)$

III. group: perioperative and postoperative cases $(\mathrm{N}=22)$ 
a similar proportion in our autopsy database [22]. We want to highlight, whoever died suddenly in Hungary, his or her autopsy was done in any case. Therefore, we got a full and accurate overview of the mortality data in this Hungarian region. Our data shows that over two thirds of ruptured cases resulted by aneurysm, while non-ruptured cases are linked predominantly to dissections. Of the non-ruptured subgroup of the AAC population, side branch occlusion occurs in about $47 \%$ of the cases $(\mathrm{N}=57)$, which is around $10 \%$ of our population $(\mathrm{N}=567)$. In published literature, we found this to be $15-40 \%[23,24]$. The low values can be explained by our choice to analyze autopsy cases only. Proving the presence of ischemia and consequential malperfusion is a difficult task even if it based on both autopsy and clinical data. The results are uncertain without the use of specialized imaging methods (MRA, CTA) or histological examination. For this reason, critical judgment and interpretation are required.

During the examined 20 year-long period (1994-2014), both the average age and the incidence increased in these two groups. Due to age distribution, at the age of $80-84$, the number of the cases increased by $48 \%$ and at the age above 85 years, the growth is $92 \%$. This would explain why we noticed a relation between the rising number of cases and age distribution. Based on these results, we believe that the examined population is an aging population. Wang et al. reported a similar tendency. They examined the autopsy reports of patients $(\mathrm{N}=909)$ who died as a result of aortic aneurysm, at the ages between 60 and 100 [22]. The results showed that AA is significantly more frequent among people at the age of 80 and above. The average age decreased only in the rAD group. This may be explained by the Central European paradox that describes an incidence increase of ever-earlier fatal cardiovascular complications (e.g. AMI) of hypertension, chronic stress and smoking [25]. The increase in the proportion of women in older age groups can be explained by the fact that women usually live 6-8 years longer [1].

Former studies reported that $60-70 \%$ of rAA cases affected the abdominal part, while $2030 \%$ affected the thoracic area, and $3-5 \%$ the thoracoabdominal area. These findings are similar to our results $[26,27]$. Earlier studies also drew attention to gender differences, although men have more aortic aneurysms but women with chest lesions are more common and the risk of rupture is greater, therefore they have a higher prehospital mortality rate. This has been explained by several molecular pathological and pathophysiological (role of oestradiol-receptor expression and circulating oestradiol-level, elastin/collagen ratio) researches, animal experiments, but further human studies are needed [28-30]. According to our findings, in the prehospital group, the mortality of rAA showed dominance at the case of thoracic ruptures, due to the fulminant progression of the disease, which causing rapid pericardial bleeding and cardiac tamponade. At the same time, the tamponading effect of the crura of the diaphragm and the retroperitoneum can cause the deceleration of the progression the abdominal ruptures.

Previous clinical observations show a two thirds to one third ratio of Stanford A and B types of aortic dissection [14], while our study shows a much higher Stanford A:B ratio (84:16). The dominance of type A dissections can be explained with the studied population, because the fulminant progression of Stanford A dissections are overrepresented in the autopsy population.

According to our study, $62 \%$ of rAD and $63.6 \%$ of rAA cases reached the hospital. Prehospital death rates for these groups were $38 \%$ and $36.4 \%$, respectively. Clinical studies of rAA showed differing results, depending on the type of aneurysm. According to Reimerink et al., 27\% of abdominal rAAA patients reached a hospital [31]. Johansson et al. reported that $47 \%$ of ruptured thoracic aortic aneurysms patients reached a hospital [32]. Our study found an approximation of $60 \%$ hospitalization rate. This result applies to the total aneurysms of the full length aorta, not just one segment, so we did an autopsy database analysis. It was difficult to compare it with the clinical data. Melvinsdottir IH et al. reported in their retrospective study that acute aortic dissection patients had a $17.6 \%$ prehospital mortality [33]. Axelsson C. et al. reported that in 2010, in Göteborg, 78\% of acute rAD patients reached an emergency department [34]. Mészáros et al. conducted a population-based longitudinal study in Hungary between 1972 and 1998 regarding the epidemiology and clinicopathology of aortic dissections. According to their study, preoperative mortality was between $21 \%$ and $79 \%$ for patients who reached a hospital [13].

We want to highlight that, in our study population, $74 \%$ of the rAA and $44 \%$ of the rAD cases reached the department of vascular or cardiac surgery. Diagnostic delays and faster $\mathrm{rAD}$ progression were difficult to separate in this research. We did not find any clinical data, it was only an autopsy database analysis.

There is a correlation with the prehospital (1), preoperative (2) and peri/postoperative (3) groups and the onset of the symptoms and the progression of the disease. In regards to the rAA cases, we reported that in the prehospital $(\mathrm{N}=110)$ and in the perioperative $(\mathrm{N}=75)$ groups, the number of ascending aorta aneurysms was significantly higher than the number of infrarenal aortic aneurysms. Bleeding into the pericardiac space after rupture was also significantly more frequent in the prehospital and preoperative groups than bleeding into the retroperitoneal space. Stanford A cases were also significantly more common in the prehospital and the preoperative groups than in the peri/postoperative group. These findings also correlate with the high percentage of pericardiac tamponade in the first two groups. It is a wellknown fact that pericardiac tamponade rapidly leads to death. In the peri/postoperative group, there were no other significant differences in the number of Stanford A and B cases. 
Our explanation for the differing results is that we analyzed an autopsy-based population. In Hungary, Emergency Departments began to function only in the past 10-15 years, while the varied clinical symptoms of aortic dissections make it harder to arrive at the correct diagnosis. There was a similar result in misdiagnosis rates in our aortic dissection population. We believe that the time factor was the answer to the question: why people with $\mathrm{rAD}$ could not reach a cardiovascular department.

\section{Conclusions}

In conclusion, we can declare that analyzing pathological data provides useful information for clinicians. It points out diagnostic mistakes, the difficulty of patient care and helps with prognostic judgment. The acute aortic dissections and thoracic aortic aneurysm ruptures are likely to lead fatal pericardial or chest bleeding. Therefore, these patients have lower chance to adequate care. Our results also show that the survival rate of chest aorta aneurysm catastrophes can be increased by rapid imaging examination (CT, MR) and an immediate surgical intervention. In cases of the abdominal aortic aneurysms a kept rupture is more frequent, which causes less cardiovascular instability, therefore more time is available to establish the diagnosis and treatment. Elective interventions have the best outcomes, so screening and planned surgeries would be considered the best treatment. From the formerly mentioned statement, a question crops up: Is the regular screening for aortic aneurysm recommended in special selected populations male, smoker, above 65 years? There is no obvious answer, either pro or con. Our results give further impetus to the sex differences in human research, as well as contribute to the development of detailed screening programs. Our research confirms that in an aging society with rising life expectancy the number of the cases is going to increase. That is the reason why we should pay attention to and promote the screening programs for patients who are exposed to risk factors or have a family history of acute aortic catastrophes. The prevention and screening is a great chance (risk factor reduction and early diagnosis) for these patients.

\section{Study Limitation}

The three institutes use different database, and they have changed several times over the 20 years. Those factors made it difficult to create a complete and consistent database. In the comparison of an autopsy-based study and a clinical-based study we have to be exact, because the examined populations are not the same just overlapping. Otherwise the data of the topic differ from each other which makes the comparison cumbersome. Our 20-year study's limitation is the examined population, we can not use correctly the concept mortality and incidence, because all our data is based upon autopsy reports.

Acknowledgements We would hereby like to thank dr. György Dunai for all the help he provided with database analysis. We would also like to thank professor Nagai Tatsuo's Pioneering Work Medal grant for the financial support.

This work was supported by the Higher Education Excellence Program of the Ministry of Human Capacities in the frame of Biotechnology research area of Budapest University of Technology and Economics (BME FIKP-BIO).

Funding Information Open access funding provided by Semmelweis University (SE).

\section{Compliance with Ethical Standards}

\section{Conflict of Interest None.}

Ethical Approval Semmelweis University regional Ethical Committee $133 / 2011$.

Open Access This article is licensed under a Creative Commons Attribution 4.0 International License, which permits use, sharing, adaptation, distribution and reproduction in any medium or format, as long as you give appropriate credit to the original author(s) and the source, provide a link to the Creative Commons licence, and indicate if changes were made. The images or other third party material in this article are included in the article's Creative Commons licence, unless indicated otherwise in a credit line to the material. If material is not included in the article's Creative Commons licence and your intended use is not permitted by statutory regulation or exceeds the permitted use, you will need to obtain permission directly from the copyright holder. To view a copy of this licence, visit http://creativecommons.org/licenses/by/4.0/.

\section{References}

1. Deaths in reference year, in Hungary (1995-2015) (2016) Hungarian Central Statistical Office

2. Clouse WD et al (1998) Improved prognosis of thoracic aortic aneurysms: a population-based study. JAMA 280(22):1926-1929

3. Upchurch GRJ (2014) Thoracic and Thoracoabdominal Aortic Aneurysms: Evaluation and Decision Making. Rutherford's Vascular Surgery, Edition E, Cronenwett JL, Johnston KW (eds), Elsevier Saunders, Philadelphia, pp 2084-2101

4. Coady MA et al (1997) What is the appropriate size criterion for resection of thoracic aortic aneurysms? J Thorac Cardiovasc Surg 113(3):476-491; discussion 489-491

5. Davies RR et al (2006) Novel measurement of relative aortic size predicts rupture of thoracic aortic aneurysms. Ann Thorac Surg 81(1):169-177

6. Kuivaniemi H, Elmore JR (2012) Opportunities in abdominal aortic aneurysm research: epidemiology, genetics, and pathophysiology. Ann Vasc Surg 26(6):862-870

7. Holt PJE, Thompson MM (2014) Abdominal aortic aneurysms: evaluation and decision making. Rutherford's Vascular Surgery, Edition E, Cronenwett JL, Johnston KW (eds), Elsevier Saunders, Philadelphia, pp 1999-2023

8. Wilmink AB, Quick CR (1998) Epidemiology and potential for prevention of abdominal aortic aneurysm. Br J Surg 85(2):155-162 
9. Robertson L, Nandhra S (2017) Laparoscopic surgery for elective abdominal aortic aneurysm repair. Cochrane Database Syst Rev 5: CD012302

10. Choksy SA, Wilmink AB, Quick CR (1999) Ruptured abdominal aortic aneurysm in the Huntingdon district: a 10-year experience. Ann R Coll Surg Engl 81(1):27-31

11. Schlosser FJ et al (2010) Mortality after elective abdominal aortic aneurysm repair. Ann Surg 251(1):158-164

12. Conrad MF, Cambria RP (2014) Aortic dissection. Rutherford's Vascular Surgery, Edition E, Cronenwett JL, Johnston KW (eds), Elsevier Saunders, Philadelphia, pp 2169-2188

13. Meszaros I et al (2000) Epidemiology and clinicopathology of aortic dissection. Chest 117(5):1271-1278

14. Hagan PG et al (2000) The International Registry of Acute Aortic Dissection (IRAD): new insights into an old disease. JAMA 283(7): 897-903

15. Januzzi JL et al (2004) Characterizing the young patient with aortic dissection: results from the International Registry of Aortic Dissection (IRAD). J Am Coll Cardiol 43(4):665-669

16. Mehta RH et al (2002) Predicting death in patients with acute type a aortic dissection. Circulation 105(2):200-206

17. Cambria RP et al (1988) Vascular complications associated with spontaneous aortic dissection. J Vasc Surg 7(2):199-209

18. Khan IA, Nair CK (2002) Clinical, diagnostic, and management perspectives of aortic dissection. Chest 122(1):311-328

19. Distribution of Hungary's population (2nd November 2017) Hungarian Central Statistical Office

20. Brindley P, Stembridge VA (1956) Aneurysms of the aorta; a clinicopathologic study of 369 necropsy cases. Am J Pathol 32(1):6782

21. Olsson $\mathrm{C}$ et al (2002) Thoracic aortic aneurysm and dissection: increasing prevalence and improved outcomes reported in a nationwide population-based study of more than 14,000 cases from 1987 to 2002. Circulation 114(24):2611-2618

22. Global regional (2016) and national incidence, prevalence, and years lived with disability for 310 diseases and injuries, 1990 2015: a systematic analysis for the Global Burden of Disease Study 2015. Lancet 388(10053):1545-1602
23. Lauterbach SR et al (2001) Contemporary management of aortic branch compromise resulting from acute aortic dissection. J Vasc Surg 33(6): 1185-1192

24. Grimm JC et al (2016) Differential outcomes of type A dissection with malperfusion according to affected organ system. Ann Cardiothorac Surg 5(3):202-208

25. Kopp MS, Rethelyi J (2004) Where psychology meets physiology: chronic stress and premature mortality - the Central-Eastern European health paradox. Brain Res Bull 62(5):351-367

26. Young R, Ostertag H (1987) Incidence, etiology and risk of rupture of aortic aneurysm. An autopsy study. Dtsch Med Wochenschr 112(33):1253-1256

27. Hoornweg LL et al (2008) Meta analysis on mortality of ruptured abdominal aortic aneurysms. Eur J Vasc Endovasc Surg 35(5):558 570

28. Makrygiannis $\mathrm{G}$ et al (2014) Sex differences in abdominal aortic aneurysm: the role of sex hormones. Ann Vasc Surg 28(8):19461958

29. Villard C et al (2011) Reproductive history in women with abdominal aortic aneurysms. J Vasc Surg 54(2):341-345

30. Villard C et al (2017) Differential expression of sex hormone receptors in abdominal aortic aneurysms. Maturitas 96:39-44

31. Reimerink JJ et al (2013) Systematic review and meta-analysis of population-based mortality from ruptured abdominal aortic aneurysm. Br J Surg 100(11):1405-1413

32. Johansson G, Markstrom U, Swedenborg J (1995) Ruptured thoracic aortic aneurysms: a study of incidence and mortality rates. J Vasc Surg 21(6):985-988

33. Melvinsdottir IH et al (2016) The incidence and mortality of acute thoracic aortic dissection: results from a whole nation study. Eur J Cardiothorac Surg 50(6):1111-1117

34. Axelsson $\mathrm{C}$ et al (2015) A description of the prehospital phase of aortic dissection in terms of early suspicion and treatment. Prehosp Disaster Med 30(2):155-162

Publisher's Note Springer Nature remains neutral with regard to jurisdictional claims in published maps and institutional affiliations. 